Monatsschrift f. Geburtshülfe u. Gynäkologie 1926;74:I-IV

\title{
Contents, Vol. 74, 1926
}

Inhaltsverzeichnis.

Originalarbeiten. Seiu

Baer, Walter, Über die Bedeutung der Blutgruppenbestimmung

fur die Bluttransfusion 284

B ähr , Sterilisation und Schwangerschaftsunterbreclrang vomRechts-

standpunkt 88

Barsky, Ch., Zur Frage über die Konstanz der Isoagglutinations-

charakteristik des Blutes $\quad 66$

Bauer, Albert W., Über Verdauungsstörungen (Atonia ventriculi

und Obstipation) als eine Hauptursache atypischer Uterus-

blutungen 165

Brandt, Heinz, liber angeborene Kniegelenkskontraktur 359

Braun, Walter, Beiträge zur Halzium-Therapie in der gynäko-

logischen Praxis unter besonderer Berücksichtigung des Hämo-

sistan 16U

Budde, Max, Über die Entstehung der Fetalfnkl·usionen, kompli-

zierten Dermoide und Teratome und ihre Beziekungen zu-

einander 276

Dick, W., Über die physiologische Richtung in der Gynäkologie.

(Kritisch-literarischer TJmriß.) 146

Dienst, Arthur, Zur Atiologie und Pathogenese der Eklampsie

und der Schwangerschaftstoxikosen 1

Esser, M., Zur Kasuistik der Uterussteine 265

Eufinger, Heinrich, Die Beeinflussung der Kolloidstabilität des

Plasma durch den monatlicken Zyklus 139

Galant, Joh. Susman, Über ein hysterisches PMnomen der

Sexualsphäre des Weibes, genannt Olobus abdominalis .... 338 fíeim, Konrad, Über menschliche Isoantikörper in Blut und Milch 52 Jackie, K., siehe Martin, Ed. Kaß, S., siehe Rosenblatt, J.

Klaî'ten, E., Zur Kenntnis der hypophysären Amenorrhö.e 38

Koerner, J., Zur Frage der Stumpftumoren 76

Liegner, B., Die Anwendung psychischer Behandlungsmethoden

in der G-ynäkologie $\quad 8(\mathrm{~J}$

Mahnert, Alfons, Über den Einfluß des Insulins auf die I-Ýörper-

gewichtskurve von frühgeborenen und lebensschwachen Kindern 347 Martin, A., und Martin, Ed., Tagesfragen. I. A. Martin, Mutter-

schaftsfragen. - II. Ed. Martin, Hebammen- und Arztefragen 103 Martin, Ed., Das preußische und das österreichische Hebammen-

gesetz 106

siehe Martin, A. 
und Jackie, K., Die Gwathmeysche Narkose 319

Offer geld, Heinr. , Über Toxikosen mit Perniziosabefund. . . . 245

JY ínhaltsverzeichnis.

Seito

Ott, D. v., Die prophylaktische mediane Dammspaltung während

der Geburt . 145

Poeck, Ernst, Über Emphysembildung bei asphyktischen Neu-

geborenen 341

Rosenblatt, J., und Kaß, S., Die ßöntgenographie als diagnosti-

sches Hilfsmittel in der Gynäkologie 182'

Scheffzek, Franz Alois, Über Uterusrupturen 34

Schneider, Georg Heinrich, Der Einfluß Immoraler Ver-

änderungen der Schwangerschaft auf das rote Blutbild .... 14 Váró, ßéla v., Über die Exstirpation

von Ovarialtumoren bei

Schwangeren 28

Weinzierl, Egon, Zur Frage der Wachstumsschnelligkeit des

Carcinoma colli uteri 322

Vereins· und Literaturbeilage.

Origínal-Sitzungsberichte aus geburtshilflich-gynäkologischen Gesellschaften:

Gynäkologische Gesellschaft in Breslau.

Sitzung vom 16. III. $1926 \quad 108$

Geburtshilflich-gynäkologische Gesellschaft in Wien.

Sitzung vom'9. III. $1926 \quad 118$

Sitzung vom 11. V. $1926 \quad 297$

Sitzungen vom 8. und 22. VI. 1926369

Die fünfzigste Tagung der Deutschen Gesellschaft für Chirurgie.

7.-10. IV. $1926 \quad 119$

Gesellschaft für Geburtshilfe und Gynäkologie in Berlin.

Sitzungen vom 12. und 26. II., 12. III., 23. IV. und 24. V. 1926 . 194

Sitzung vom 12. VI. $1926 \quad 294$

Nordostdeutsche Gesellschaft für Gynäkologie.

Sitzung vom 27. II. $1926 \quad 212$

Sitzung am 12. V. 1926 in Danzig 363

Gemeinsame Sitzung der Ober-. Mittel- und Niederrheinisch-West-

fälischen Gesellschaft für Geburtshilfe und Gynäkologie am

13. VI. 1926 in Bad Kreuznach “ 298

Referaíe aus geburtshilflich-gynäk. Gesellschaften 124, 215, 301, 371 Geburtshilflich-

gynäkologische Mîtteilungen aus anderen medi-zinischen Gesellschaften und Vereinen . . 125,

216, 302, 372.

Buchbesprechungen 130, 220, 304, 37a

üteraturverzeichnis 133, 230, 309, 376

Personalíen und Tagesnachrichten .... 138, 244, 318, 380

Druckfehlerverzeíchnís 380

Hans Thaler $† 317$

Karl Pranz $\dagger 381$

Erich Opitz $\dagger$ 388- 